\title{
Assessment of Combinatorial Support Assemblies and Their Energy Matrixes in High Tension Bolt Couplings
}

\author{
E. E. Jumbo, P. P. Jombo, E. M. Adigio \\ Department of Mechanical/ Marine Engineering, Niger Delta University, Wilberforce Island, Bayelsa State, \\ Nigeria.
}

\begin{abstract}
The bolt and nut coupling are fundamental design requirement for machines and steel structures and their relevance and utilization are of topmost priority in the areas of their application. The paper viewed the component parts and dimensions of this coupling from the perspective of energy content delivery, balancing and equilibrium. This result from the fact that the supply of compression torque on the coupling converts ingrained residual energy in the individual parts into utility energy assets for the support and sustenance of structures of interest. The mechanics and practicality of this energy matrix theory has been investigated using congruent scientific analysis, conventional tables, lab test data and graphics which depicts boundary behavioral tendencies resulting from structural realignments in the face of increasing load value. The extreme limit of this realignments, culminate in varying degrees of yield conditions particular viewed as deformation, which occurs at the point of lowest energy availability in the coupled system.
\end{abstract}

Keyword: geometryof forces, shear stress, bolt preload, energy relativity, shear off, ultimate value shearing stresses, crushing stress, ingrained limits, synergetic value, micro-synergy centers

\section{Introduction}

Bolts and nuts are coupling members with characteristic attributes that jointly results the capability of fastening and work holding of machine pieces together. A view of Figure 1 below, indicates the presence of the following functional members forming the coupling. They include:

(i) thread (ii) nut (iii) runout (iv) shank (v) radius (vi) head

A combination of these 6-in- 1 coupling also indicates:

(a) thread length - runout to initial root diameter

(b) grip length - runout to shank radius

(c) nominal length - shank radius to initial root diameter.

In view of the foregoing, the aim of this paper is to identify the contributory forces that are supplied by these functional members; analyze their interface relationship in view of their combinatorial support factors and assess how this support translates into energy regimes required to balance the tensional stress that sustains the torque on the bolt-nut engagement.

The applicability of this regime depends largely on the bolt and nut geometry and how their interrelationship with the applied torque ensures or results frictional forces (tangential, axial, kinetic, etc.) which exist in the coupling as balancing and binding energies. Further, in order to retain this accumulated energy, the geometrical and helical orientations of the thread, (indicating a series of equilateral triangles with chopped off crest and rounded out roots), are intentionally designed to ensure structural support for the accumulation and transmission of the energy resulting from the interrelationship and interactions of the frictional forces and process variables.

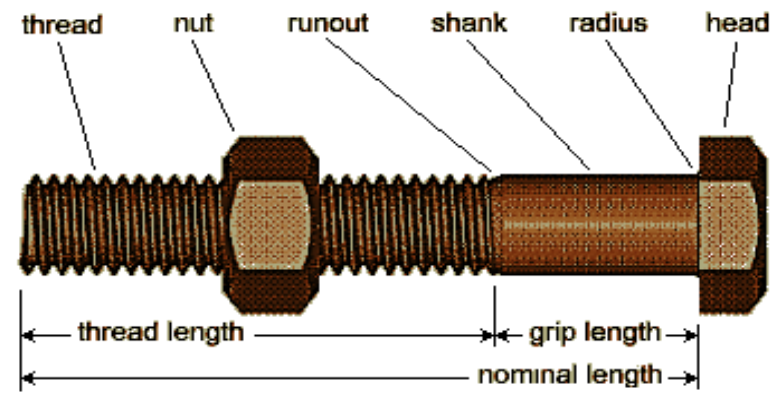

Figure 1- bolt and nut assembly

Courtesy AME-Lab, Niger Delta University 
A better understanding of this geometryof forces rest in the fact that in a typical design for bolt, the machinist or manufacturer maintains cutting parameters which guarantees that the depth of the threads is $54.127 \%$ of the distance between the threads and the radius of the rounded root is $14.434 \%$ of the distance between threads. It should be noted that this geometrical orientation constitutes and enhances energy sinks and reservoirs; for which the structural integrity of the aforementioned dimensions imply a consequential support. Further, as Figure 2 indicates, $1 / 8$ of the height of each of the equilateral triangle is cut off at the top; and at the bottom 5/8 is available after cutting off $1 / 4$ of the height of the bottom. These dimensions are conventionally necessary to ensure efficient energy accumulation and transmission in the system.

This paper is of the firm view that these design considerations are necessary for the accumulation and transmission of energy generated from engaged frictional forces. This position is further reinforced in the fact that the root diameter of the thread is the nominal diameter less $108.3 \%$ of the pitch of the thread. The practical implication of this geometrical arrangement is that fine threads have large root diameters than coarse threads and as such accumulates and transmits more energy [1].

Further, at the heart of this interaction of frictional forces is the view that threads in a bolt do not absorb equal share of the load [1].This fortifies the possibility that one cardinal thread can actually assume the total load capacity and only transmit marginal or residual load $W_{R}$ to other threads upon attainment of initial boundary limit tending towards material yielding stress, $\boldsymbol{\tau}_{Y}$ condition. However, on the other hand if we assume that the load is evenly distributed among the threads, then such engagement in the view of this paper should be dependent on the geometrical process variable as defined by the following expressions, which results from the assumption that the external load which acts a force, tangential to the surface of the contact points of the bolt and nuts would not result a deformation of the elementary volume or length of bolts or nuts. This assumption clearly requires that both shear stress on the bolt and nut expressed as:

$\tau_{(\text {nut })}=\underline{F}$

$\pi d(h / 2)$

and

$\boldsymbol{\tau}_{(b o l t)}=\underline{F}$

$\pi d_{r}(h / 2)$

must be resolved together with the bearing stress between the threads, expressed as:

\begin{tabular}{|c|c|c|}
\hline \multicolumn{3}{|c|}{$\begin{array}{l}\sigma_{b}=\quad \underline{F} \\
\pi / 4\left(d^{2}-d_{r}^{2}\right)(h / p) \ldots \ldots \ldots \ldots \ldots \ldots \ldots(3) \\
\text { Where: }\end{array}$} \\
\hline & $=$ & shear stress \\
\hline & $=$ & bearing stress \\
\hline & $=$ & outside diameter of bolt \\
\hline & $=$ & height of nut \\
\hline & $=$ & root diameter of bolt an \\
\hline & $=$ & pitch of bolt \\
\hline
\end{tabular}

In view of the assertions in equations (1) to (3), it is safe to posit that while forces both normal, resistive and tangential, interact among the component parts of the bolt-nut coupling, their combinatorial consequence is power build-up, which is stored at the contact points of the bolt and nuts interface and when cumulatively considered among the interacting members constituting the coupling (i.e. bolt, nut and assembly), results a binding energy stored in the form of potential energy. Thus, this energy is the result of the combinatorial relationship and interactions [2] i.e. energy transfer and distribution among the component parts identified in Figure 1 above.

\section{Load - Stress Relativity And The Energy Factor}

It has been reported that stress is an internal distributed force and thus constitutes the internal mechanical reaction of the impacted material to an external force just before deformation [2]. In line with this position, it should be pointed that if stress is a reactionary consequence of an external or internal force condition, then it must occur in pairs; namely:

1) Normal stress, defined as;

(i) tensile stress

(ii) compressive stress 
2) Tangential or shearing stress.

Pursuant to the foregoing argument, when torque (tightening force) is applied to a bolt in axial direction of the thread, the nut constitute an internal stress (force) that must be resisted by the bolt. The observed resistance to the free movement of the nut is referred to, as bolt stiffness, $\mathrm{E}=$ stress/strain or $\sigma / \mathrm{e}$. Thus, it could be held that this stiffness connotes the presence of a load on the bolt and this load is a factorial function of normal and tangential forces of the threads of the nut on the threads of the bolt, subsumed as the impact force for which a resistive force (i.e. stress) arises to balance of the impacted force and thus maintains an equilibrium of forces, as indicated in Figure 2 below.

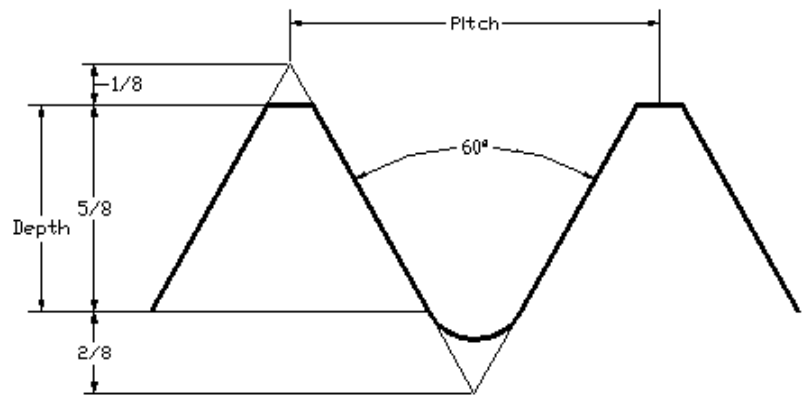

Figure 2- thread formation geometry indicating energy sink as projecting triangles on thread top and base

As could be seen in the figure above, the nut constitutes an internal load to the bolt through the interface of the bolt thread engaged in the nut thread. Though not explicit in Figure two above, there exist a clearance zone along the interlacing thick threadline interfacing between the bolt and nut. It should be noted that the existence of the cut-off at the crest of both threads creates power interchange resulting from both compressive and attendant resistive forces, of the threads.

This condition where a nut places tension on a bolt is called bolt preload, which should exceed the maximum load by $15 \%$ based on standard industry practice. This paper is off the view that this is only possible where the joint is stiffer than the bolt. Further, it should be a design consideration that the shank of high tension bolt should be of the same diameter to the root of the thread [2]. This implies that energy relativity exists between the load and the corresponding stress on the bolt with respect to the sizes of the shank in relation to the root of the bolt.

This geometrical reality connotes that, since shank diameter is equal to root diameter of the thread, they are both of equal strength and none is weaker than the other. This adds up to the fact that bolt strength is evenly distributed along the bolt-shank axis and further means that on the event of failure, either point on the axis could be affected depending on the direct position of the external load.

As could be shown in the table below:

Table 1- American/United National Threads

\begin{tabular}{|c|c|c|c|l|}
\hline Size & Diameter & $\begin{array}{l}\text { TPI } \\
\text { Coarse }\end{array}$ & $\begin{array}{l}\text { TPI } \\
\text { Fine }\end{array}$ & $\begin{array}{l}\text { Root } \\
\text { Dia. } \\
\text { Coarse }\end{array}$ \\
\hline$\# 0$ & 0.0600 & - & 80 & 0.0447 \\
\hline$\# 1$ & 0.0730 & 64 & 72 & 0.0560 \\
\hline$\# 2$ & 0.0860 & 56 & 64 & 0.0668 \\
\hline$\# 3$ & 0.0990 & 48 & 56 & 0.0771 \\
\hline$\# 4$ & 0.1120 & 40 & 48 & 0.0813 \\
\hline$\# 5$ & 0.1250 & 40 & 44 & 0.0971 \\
\hline$\# 6$ & 0.1380 & 32 & 40 & 0.1073 \\
\hline$\# 8$ & 0.1640 & 32 & 36 & 0.1299 \\
\hline$\# 10$ & 0.1900 & 24 & 32 & 0.1570 \\
\hline$\# 12$ & 0.2160 & 24 & 28 & 0.1722 \\
\hline
\end{tabular}


The unified system of threads pursuant to NATO 1948 standardization, resolved the technical dichotomy between American bolts (with flat bottom groves in between threads), which interfered with British roundtopped threads. It should be noted that before the 1948 stipulation, British bolts could not fit in with American nuts. Thus, the unified system resolved these differences. Also, the table show varying percentage of differentials in diameter between the shank diameter and the root diameter.

Thus, implying that a difference in load bearing capacity between the shank and the root diameter exist as a critical design factor with energy relativity connotation. Based on this position, it can be said that a bolt's failure point lies to the root diameter. Thus, the energy factor in this circumstance is directly derivable from the geometrical interplay between frictional forces on the bolt thread and those on the nut.

\section{Process Variables And Resultant Energy Matrices-}

Relativity In The Design Of Bolts And Nut Couplings

Energy matrices as viewed by this paper have to do with design parameters which form the bases of the construction of bolts and nuts. These parameters are implemented as process variables that could be manipulated to achieve design specifications. Thus, at the point of design calculations, load support and distribution (as stated earlier in this paper) are the major factors of concern; as this support and distribution are unconsciously assigned to the various variables. The limits of these variables thus determine the extent of the design and the possible range of loads the design can support.

It is therefore insightful to state that during design considerations, the load value distribution network, linkage and support structures represent energy flow lines; and a structural arrangement of these flowlines could be viewed as energy matrices intended to support and sustain the process variables. In the sections that follow the paper shall state the parameters and conditionalities required to achieve this energy storage and transmission.

\subsection{Process Variable and Design Interface}

In Figure 3 below, the core diameter; $d_{i}$, major diameter, $d$ and pitch $p$ are shown along-side the thread which is the subject of determination for energy balance based on the axial load value and utilization, since the load comes on the bolt as an axial force.

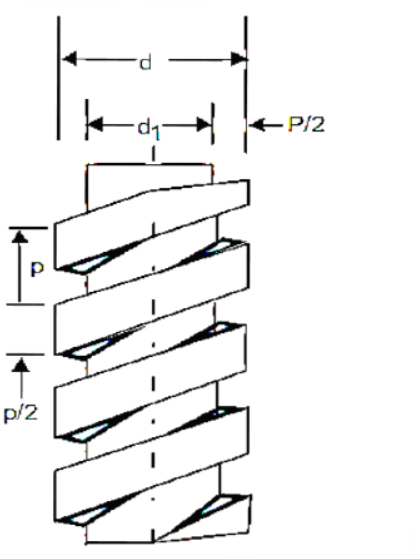

Figure 3 -structural composition of a bolt indicating applicable process variables

Thus, the application of load, $W$ on the coupling, impact a compression resulting a compressive stress distributed cross-sectionally on $d_{l}$. Under the rotating application of a wrench, the bolt also rotates, thus subjecting the nut to a torque, resolved as

$\mathrm{P} \underline{\mathrm{d}}_{\underline{\mathrm{m}}}$

Where geometrically positioning of forces are required, equation (4) can be represented as an energy quantization factor, wherein the presence of $W$ results a force to force reciprocation, where compressive forces are resisted and a system of energy accumulation and dissipation is established; thus creating a supporting balance, for which tangential (tan $\alpha$ )and axial ( $\tan \varphi)$ forces interplay within the boundary mean $\left(d_{m}\right)$ of $d$ and $d_{1}$ in the following expression;

$W=\tan (\alpha+\varphi) \underline{d}_{\underline{m}}$

In both cases: 
$P=$ effort from the wrench

$d_{m}=$ mean diameter (i.e. $\frac{d+d_{1}}{2}$ )

$W=$ weight of the bolt on action of $P$

$\tan \alpha=$ angle of helix (helical pitch orientation w.r.t. $P$ )

$\tan \varphi=$ angle of friction

It should be noted, that equation (4) constitutes a torque, $\tau$ on the bolt and would result a shearing stress which has been defined as "the ratio of the applied shearing force to the cross-sectional area being sheared [3]. Thus, $\boldsymbol{\tau}$ would have maximum value at the surface of bolt, defined at the point $\mathrm{d}_{1} / 2$, which also represent the position of highest axial load value between the threads of the bolt and the nut.

Consequently, wrench effort $P$, which results pressure and partial loading on the nut should not exceed permissible limits as conventionally required in standard tables and operational guidelines for specific couplings. This position results from the fact that upon increased value of $P$, the thread and cylindrical surface of the bolt or nut (depending on which is lesser in strength) may tend to shear off or fail under the load acting on the thread.

\subsection{Energy Content Delivery by Direct Compressive Stress}

Deducible inference from equation (5) indicate that the axial load $W$, which by nature is a direct compressive force impacted on the core cross-section of diameter $d_{l}$, results an energy content factor, defined as compressive stress and expressed as;

$$
\begin{aligned}
& \sigma=4 \mathrm{~W} \\
& \pi \mathrm{d}_{1}{ }^{2}
\end{aligned}
$$

It should be noted that the importance of this quantity rests in its ability to coexist and combine with shearing stress to result the ultimate value shearing stresses to which the coupling is subjected. Further, the complexity of this energy conduction requires the utilization of the given value of $\sigma$ in the determination of the minor diameter $d_{l}$. This implies that permissible compressive stress is determinable from the design limits of the load carrying capacity of $d_{l}$ and in view of this condition the magnitude of compressive stress has to be increased by $30 \%$ energy content, hence resulting the expression.

$$
\sigma=\frac{1.3 \mathrm{~W}}{\underline{\pi \mathrm{d}}_{1}^{2}}
$$

4

Worthy of mention is the fact that the satisfaction of equation (7) is the deterministic conditionality for the

\begin{tabular}{|c|c|c|c|c|c|c|c|}
\hline \multirow[t]{2}{*}{$\begin{array}{l}\text { Design } \\
\text { ation }\end{array}$} & \multirow[t]{2}{*}{$\begin{array}{l}p \\
\mathbf{m m}\end{array}$} & \multirow[t]{2}{*}{$\begin{array}{c}\mathbf{d} \text { or } D \\
\mathbf{m m}\end{array}$} & \multirow[t]{2}{*}{$\underset{\mathbf{m m}}{\mathbf{d}_{\mathbf{p}}}$} & \multirow{2}{*}{\begin{tabular}{|c|}
$\begin{array}{c}\mathrm{D}_{\mathrm{c}} \\
\mathbf{m m}\end{array}$ \\
Nut \\
\end{tabular}} & \multirow{2}{*}{$\begin{array}{r}\begin{array}{r}D_{c} \\
\text { mm }\end{array} \\
\text { Bolt }\end{array}$} & \multirow[t]{2}{*}{$\begin{array}{l}\text { Thread } \\
\text { Depth } \\
\text { mm }\end{array}$} & \multirow[t]{2}{*}{$\begin{array}{l}\text { Stress } \\
\text { Area } \\
\text { mm }^{2}\end{array}$} \\
\hline & & & & & & & \\
\hline M10 & 1.5 & 10.000 & 9.026 & 8.876 & $\begin{array}{l}8.16 \\
0\end{array}$ & 0.920 & 58.30 \\
\hline M12 & 1.75 & 12.000 & 10.863 & $\begin{array}{l}10.10 \\
6 \\
\end{array}$ & $\begin{array}{l}9.85 \\
8 \\
\end{array}$ & 1.074 & 84.00 \\
\hline M14 & 2 & 14.000 & 12.701 & $\begin{array}{l}11.83 \\
6\end{array}$ & $\begin{array}{l}11.5 \\
64 \\
\end{array}$ & 1.227 & 115.00 \\
\hline M16 & 2 & 16.000 & 14.701 & $\begin{array}{l}13.89 \\
8\end{array}$ & $\begin{array}{l}13.5 \\
45\end{array}$ & 1.227 & 157.00 \\
\hline M18 & 2.5 & 18.000 & 16.376 & $\begin{array}{l}15.29 \\
4\end{array}$ & $\begin{array}{l}14.9 \\
33 \\
\end{array}$ & 1.534 & 192 \\
\hline M20 & 2.5 & 20.000 & 18.376 & $\begin{array}{l}17.29 \\
4\end{array}$ & $\begin{array}{l}16.9 \\
33\end{array}$ & 1.534 & 245 \\
\hline M24 & 3 & 24.000 & 22.051 & $\begin{array}{l}20.75 \\
2 \\
\end{array}$ & $\begin{array}{l}20.3 \\
20 \\
\end{array}$ & 1.840 & 353 \\
\hline M30 & 3.5 & 30.000 & 27.727 & $\begin{array}{l}26.21 \\
1\end{array}$ & $\begin{array}{l}25.7 \\
06 \\
\end{array}$ & 2147 & 361 \\
\hline
\end{tabular}
theoretical and technical range of safe bearing pressure (in $\mathrm{MPa}$ ), in the various bolt and nut combinations in terms materials requirements and constraints, as table 2 below indicates:

Table 2-Dimensions of M10 - M30 V-Threads (Coarse Specification) indicating process variables and conditionalities.

In view of the foregoing, the entries in Table 2 reinforces the position that safe bearing pressure also defined as the crushing stress between the surface of the bolt thread and the matching contact surface of the nut thread is a definitive and measurable quantity [1]. This quantity can be expressed as; 
$\sigma_{\mathrm{B}}=\underline{\mathrm{W}}$

Where:

$\sigma_{B}=$ bearing pressure (i.e. crushing stress of projected

thread area in Pascal)

$W=$ load

$d_{m}=$ mean screw thread diameter

$h=$ depth of thread

$n=$ number of threads in engagement

In view of the relevance of equation (8), it is in order to point that a force distribution line as stated earlier exists which maps all the frictional component points in the bolt-nut coupling into a power distribution network of energy lines looking much like a load connection matrix. These energy lines maintain a safe limit of sustenance above or beyond which the weaker point of the coupling would fail, resulting damage and deformation of the coupling.

The foregoing implies that in all machineries and structures sustained by bolt and nut couplings, a network of energy profile is established within the ingrained limits of the materials' crystalline configuration. Thus, the positions of these couplings are by the averments of this paper, energy quantization and characterization points which collectively establish aggregate equilibrium balance of synergetic value which is only distorted upon failure resulting from tendencies attributable to violations of safe bearing pressure limits.

Accordingly, all the component parts indicated in Figure 1 above contribute to the overall safe bearing pressure of the coupling, since the identified parts are micro-synergy centers within the energy matrix.

\subsection{Energy Content Delivery by Maximum Shearing Stress Quantization}

As pointed earlier, equation (5) is an energy quantization factor with significant tangential and axial force proclivity; capable of causing a rotation of the load $W$ (i.e. bolt) in the direction of effort $P$ (wrench). This characteristic inertia overcomes all potential, residual and incidental forces on and against $W$ and subjects it to the control of work variables which do not only convert these identified forces to "measurable energy quanta", but also engages them as capitals for a constructive power reservoir under a holding capacity that balances all direct and incidental forces within the known design limits.

Further, equation (5) is not only an inertia factor but also a shearing stress initiator analogous to the position in section 2.2 above. In view of this situation, the shearing stress component of this energy content delivery, directly relate to the ratio between the torque element on $W$ and the distance $\pi d$, covered.

This implies that the transmission of energy on $W$ by the action of $P$, also delivers energy along the spiral line of the helix on the bolt-nut interface. The implication of this finding is that equation (5) results a shearing stress condition on the coupling which could be defined as;

$$
\tau=\frac{16 M_{t}}{\pi d_{1}^{3}}
$$

Where $M_{t}$ is a resultant tangential moment to, and about $W$ and defined by $P d_{m} / 2$.

In the foregoing argument, the energy state of the coupling becomes a 3-dimensional cubic co-factor energy combination incumbent on the values of $\sigma$ and $\tau$ as Figure 4 below indicates the energy build-up structure as follows:

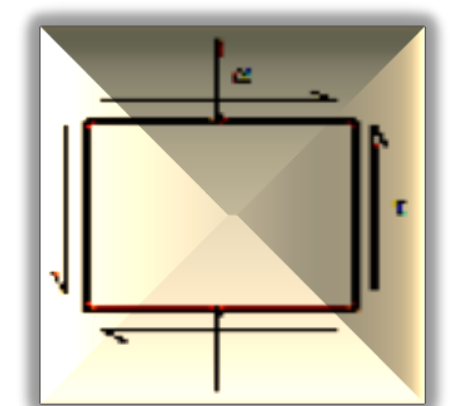

Figure 4 Cubic co-factor force combination indicating process interplay between compressive stress, $\sigma$ and shearing stress, $\tau$; thus giving rise to energy build-up in direct proportion to design constraints of the coupling. As could be seen, the pointed tip defines the upper limits of load capacity beyond which a failure results 
It should be noted that this combination results the cubic orientation of the root diameter $d_{1}$ giving rise to the above figure. Pursuant to Figure 4 above, researchers have viewed this $\sigma$ and $\tau$ interplay as an even distribution of forces within the surface of core of diameter $d_{l}$ [4]. The position of this paper is that since the increasing action of $P$ on $W$ implies increased energy delivery to the coupling, the resultant $\sigma . \tau$ forces combination in the system would also respond to the energy build-up profile in such a graduated manner that, increase of $P$ on $W$ causes an increase in the combine value of $\sigma . \tau$, and if allowed can progress to the extreme limit beyond which the weaker member of the coupling would yield, irrespective of the strength of the other. This is practically experienced in situations where the bolt and nut are made of different materials with different strength profiles.

In line with the foregoing, it is thus reasonable to posit that Figure 4 is a direct consequence of effort $P$ on load $W$ for which the combined energy content delivery factors of $\sigma$ and $\tau$ responds with increasing magnitude, and attains maximum just before a yield, should $P$ increase above the required limit. Further, shearing stress quantization is a measurable factor that derives its meaning and value from the maximum principal stress which is expressed as;

$\sigma=\sigma / 2+\sqrt{ }(\sigma / 2)^{2}+\tau^{2}$

Further, it is instructive to state that in the calculation for permissible value of shear stress as to determine design limits, equation (10) is not of much significance because it has a reducing value upon the identified force build up (or increase of $P$ on $W$ ). Secondly, at certain points, maximum principal stress may become tensile and as such a non-contributory factor to the energy value of the coupling. Notwithstanding this condition, the maximum shearing stress is significant in the sense that;

$\tau_{\max }=\sqrt{ }(\sigma / 2)^{2}+\tau^{2}$

and when expanded equation (11) becomes:

$=\sqrt{ }\left(4 \mathrm{~W} / 2 \pi \mathrm{d}_{1}^{2}\right)^{2}+\left(16 \mathrm{M}_{\mathrm{t}} / \pi \mathrm{d}_{1}^{3}\right)^{2}$

or;

$\tau_{\max }=16 / \pi \sqrt{ } W^{2} / 64 d_{l}^{4}+M^{2} / d_{l}^{6}$

Thus, a solution to equation (12) yields the permissible shearing stress. However, this solution is difficult in practical terms due to the polynomial condition of $d_{1}$ which geometrically progresses up to the $4^{\text {th }}$ and $6^{\text {th }}$ power. It is therefore recommended that the value of $d_{l}$ be determined from equation (7), before resolving equation (12) to solve for $\tau_{\max }$. When this is done, it would be seen that $\tau_{\max }$ value is less than permissible value of shearing stress.

\section{Discussions}

The consequential energy containment and dissipation relevance of equation (12) connotes the possibility that, for a bolt and nut coupling to serve as energy reservoir to sustain a load or perform a defined function, then a maximum shearing stress is required (though it constitute a minimum force). Secondly, this minimal force must be less than the permissible values of shearing stress required to contain or transmit the energy generated or stored. This matrix of energy accumulation and dissipation by the action of $P$ on $W$ under the combined limits of $\sigma$ and $\tau$ are thus governed by process variables involved in the interrelationships of the earlier identified energy centers in Figure 1.

Central to the averments above, is the fact that although bolts are made of steel having carbon percentages varying between 0.08 and 0.25 [3], high tension bolts as discussed in this paper are of high strength capability and made of alloy steel, with significant treatment of quenching and tempering. This heat treatment thus reinforces the crystalline structures and configurations of the combining alloying elements and this results energy matrices with established network of load bearing lines, through which force is absorbed or transmitted.

Further, it has been submitted that the force induced by action of $P$ on $W$ imposes higher stress in the bolt even without external load. Studies have shown that such stress are higher in the case of smaller diameter bolts and reduces as the size of bolt increases. Thus, a correlation is established between bolt size (that is minor diameter) and permissible stress for which a reiterative selection process is required. In order to overcome this difficulty, an empirical formula as expressed below could be utilized. Thus; permissible tensile stress, $\sigma_{t}$ and bolt diameter at the section of stress impact becomes;

$\sigma_{t}=5.375\left(d_{1}\right)^{0.84}$ 
Apply equation (13) to generate a graphic solution on a 1:20 (y-axis) and 1:5 (x-axis), the permissible stress of medium carbon steel bolt can be determined using the following table of test values, conducted at the Advance Manufacturing Engineering Laboratory, (AME-Lab) of the Niger Delta University.

Table 3 - Test data for permissible stress

\begin{tabular}{|l|l|l|l|l|l|l|l|l|l|}
\hline $\begin{array}{l}\text { Minor bolt diameter } \mathrm{d}_{1} \\
(\mathrm{~mm})\end{array}$ & 5 & 10 & 15 & 20 & 25 & 30 & 35 & 40 & 45 \\
\hline Permissible stress $(\mathrm{MPa})$ & 20 & 40 & 60 & 80 & 100 & 120 & 140 & 160 & 180 \\
\hline
\end{tabular}

Figure 5 Permissible bolt stress as function of minor core diameter $d_{l}$

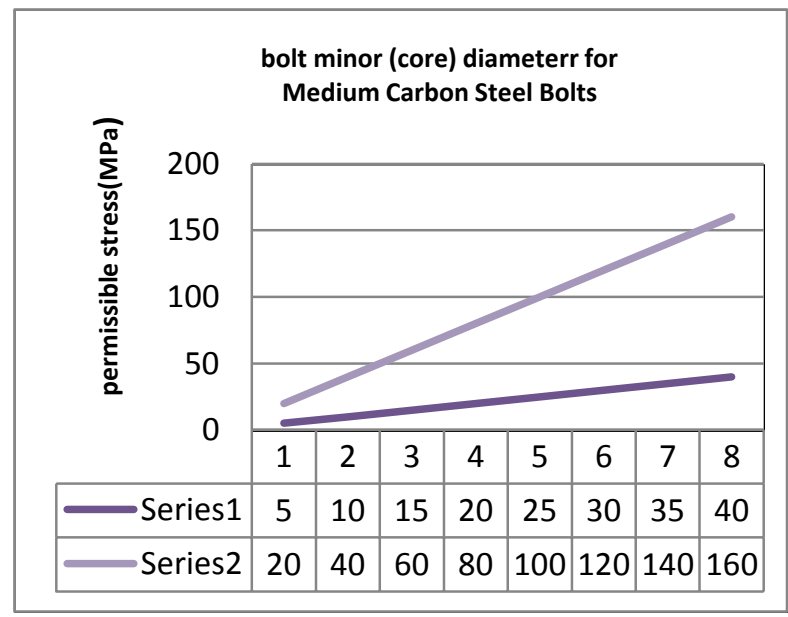

In conclusion of the findings of this paper the study has posited and sustained the argument that only energy can keep or give energy, and the bolt and nut coupling established this phenomenon in the sense that all the component parts functions as energy collection and reservoir points and these points collectively form an energy content matrix, directly dependent on the structural orientation of the crystalline formations of the metal or alloying elements, which occurs as criss-crossing lines in orderly forms representing energy storage, conduction and dissipation lines; and thus sustains or transmits energy depending on system design requirement.

\section{References}

[1]. Deutschman A.D., Michels W.J, Wilson C.E.: Machines Design- Theory and Practice, macmillian, New York, 1975, p.807-810

[2]. Vidosic, J.P, Mechanics of Material section contribution in Mark's handbook for Mechanical Engineers, Edited by Eugene A. Avalone and Theodore Baumeister III, $9^{\text {th }}$ Edition, McGraw-Hill, New York, 1978, p 5-16-5118

[3]. Kalpakjian S., Scmid S.R., Manufacturing Engineering and Technology, $4^{\text {th }}$ Edition, Pearson Education, New Jersey, 2005 , p.62

[4]. Baldwin G., Metalworking, Moldmaking and Machine Design, series contribution in Manufacturing Engineering Handbook, McGrawHill, NY, 2004, p27.10-27.29 